\title{
Discovery potential of multiton xenon detectors in neutrino electromagnetic properties
}

\author{
Chung-Chun Hsieh, ${ }^{1}$ Lakhwinder Singh, ${ }^{2,3}$ Chih-Pan Wu, ${ }^{1}$ Jiunn-Wei Chen, ${ }^{1,4,}{ }^{*}$ Hsin-Chang Chi, ${ }^{5}$ \\ C.-P. Liu $\odot,{ }^{5, \dagger}$ Mukesh K. Pandey, ${ }^{1}$ and Henry T. Wong ${ }^{2}$ \\ ${ }^{1}$ Department of Physics, Center for Theoretical Physics, and Leung Center for Cosmology and Particle \\ Astrophysics, National Taiwan University, Taipei 106, Taiwan \\ ${ }^{2}$ Institute of Physics, Academia Sinica, Taipei 11529, Taiwan \\ ${ }^{3}$ Department of Physics, Banaras Hindu University, Varanasi 221005, India \\ ${ }^{4}$ Center for Theoretical Physics, Massachusetts Institute of Technology, \\ Cambridge, Massachusetts 02139, USA \\ ${ }^{5}$ Department of Physics, National Dong Hwa University, Shoufeng, Hualien 97401, Taiwan
}

(Received 22 March 2019; published 3 October 2019)

\begin{abstract}
Next-generation xenon detectors with multiton-year exposure are powerful direct probes of dark matter candidates, in particular the favorite weakly interacting massive particles. Coupled with the features of low thresholds and backgrounds, they are also excellent telescopes of solar neutrinos. In this paper, we study the discovery potential of ton-scale xenon detectors in electromagnetic moments of solar neutrinos. Relevant neutrino-atom scattering processes are calculated by applying a state-of-the-art atomic manybody method-relativistic random phase approximation. Limits on these moments are derived from existing data and estimated with future experiment specifications. With one ton-year exposure, XENON-1T can improve the effective millicharge constraint by a factor of 2. With LZ and DARWIN, the projected improvement on the solar neutrino effective millicharge (magnetic moment) is around 7 (2) times smaller than the current bound. If LZ can keep the same background level and push the electron recoil threshold to $0.5 \mathrm{keV}$, the projected improvement on the millicharge (magnetic moment) is about 10 (3) times smaller than the current bound. An unconventional setup of placing a strong ${ }^{51} \mathrm{Cr}$ neutrino source by a ton-scale xenon detector is also considered.
\end{abstract}

DOI: $10.1103 /$ PhysRevD.100.073001

\section{INTRODUCTION}

Xenon detectors play a dominant role in direct experimental searches of a favorite dark matter candidateweakly interacting massive particles (WIMPs). The current best limits on the spin-independent WIMP-nucleon cross section are set by xenon detectors with $4.1 \times 10^{-47} \mathrm{~cm}^{2}$ for a $30 \mathrm{GeV} / c^{2}$ WIMP and $8.6 \times 10^{-47} \mathrm{~cm}^{2}$ for a $50 \mathrm{GeV} / c^{2}$ WIMP set by XENON1T [1] and PandaX-II [2] with subton-year exposure. It is expected that the nextgeneration experiments, XENONnT [3], LZ [4], and DARWIN [5], with multiton-year exposure will bring further improvement by 1 or 2 orders of magnitude.

\footnotetext{
jwc@phys.ntu.edu.tw cpliu@mail.ndhu.edu.tw
}

Published by the American Physical Society under the terms of the Creative Commons Attribution 4.0 International license. Further distribution of this work must maintain attribution to the author(s) and the published article's title, journal citation, and DOI. Funded by SCOAP ${ }^{3}$.
To achieve extremely low background, WIMP detectors are typically hosted in deep underground laboratories to shield cosmic rays. However, neutrinos from the Sun, supernovae and their remnants, and atmospheric showers can still reach the detectors to generate scattering events indistinguishable from the WIMP signals and form the socalled irreducible background. In the sub-keV to $100 \mathrm{keV}$ recoil energy that WIMP searches focus on, neutrinonucleus coherent scattering and neutrino induced atomic ionization are the main sources of background and are studied in Refs. [6] and [7,8], respectively.

This feature implies that those ton-scale, low background xenon WIMP detectors can serve as excellent solar neutrino telescopes to study solar and neutrino physics at the same time. For example, the very low energy solar neutrino flux from proton-proton fusion can be measured to the $1 \%$-level precision [7], which provides an experimental check to the standard solar model. The high energy solar neutrino flux from the ${ }^{8} \mathrm{~B}$ decays can trigger observable coherent neutrino-nucleus scattering.

In this paper, we attempt to address the discovery potential of using ton-scale liquid xenon detectors to probe 
exotic electromagnetic (EM) properties of solar neutrinos. In the Standard Model (SM) of particle physics, neutrinos are charge neutral and have extremely tiny EM moments including magnetic dipoles, electric dipoles, anapoles, and charge radii through radiative corrections. Those SM EM properties are not detectable with current experimental sensitivity but new physics beyond SM might make them detectable. Hence the detection of those exotic EM properties are important probes of new physics which could have profound implications to particle physics and astrophysics (see, e.g., Refs. $[9,10]$ for recent reviews of this topic).

The current best limits reported by direct laboratory searches for "effective" (to be explained in the next section) neutrino EM moments include the following: the magnetic moment of reactor antielectron neutrinos $\mu_{\bar{\nu}_{e}}^{\text {eff }}<2.9 \times 10^{-11} \mu_{\mathrm{B}}\left(\mu_{\mathrm{B}}\right.$ is the Bohr magneton) by the GEMMA experiment using a germanium detector [11]; the magnetic moment of solar neutrinos $\mu_{\nu_{\mathrm{S}}}^{\text {eff }}<2.8 \times 10^{-11} \mu_{\mathrm{B}}$ by the Borexino experiment using a liquid scintillator [12]; the effective millicharge of reactor antielectron neutrinos $\delta_{\bar{\nu}_{e}}^{\text {eff }}<1.5 \times 10^{-12} e_{0}\left(e_{0}\right.$ is the positron charge) using data from the GEMMA experiment [13], and $\delta_{\bar{\nu}_{e}}^{\text {eff }}<$ $2.1 \times 10^{-12} e_{0}$ by the TEXONO experiment using a smaller germanium detector with a lower threshold than the one of GEMMA [14]; and the effective charge radius squared of reactor antielectron neutrinos $\left\langle r_{\bar{\nu}_{e}}^{2}\right\rangle^{\text {eff }}<$ $3.3 \times 10^{-32} \mathrm{~cm}^{2}$ by the TEXONO experiment using a CsI scintillator [15]. Our main goal of this study is to quantify whether these limits can be improved with xenon detectors with multiton-year exposure to solar neutrinos using realistic detector specifications of energy threshold, energy resolution, and background.

To achieve this goal, we need to calculate the scattering cross sections of solar neutrinos and xenon atoms, and use them to predict the event rates expected at detectors. Because the energy scales in these scattering processes, which range from a few tens of $\mathrm{eV}$ to a few tens of $\mathrm{keV}$, overlap with the ones of atomic physics, it is necessary to perform reliable many-body computations. Similar to our previous works on neutrino-germanium scattering [14,16,17], we apply an $a b$ initio approach, the relativistic random phase approximation (RRPA) [18-21], to neutrino-xenon scattering. We report new results of neutrino-xenon scattering through exotic EM interactions, together with the weak interaction process calculated in Ref. [8] for completeness.

\section{SCATTERING OF SOLAR NEUTRINOS OFF XENON ATOMS}

In the most general case, the EM current of a neutrino field, $\nu$, is given by

$$
\begin{aligned}
j_{\mu}^{(\gamma)}= & \bar{\nu}\left[F_{1}\left(q^{2}\right) \gamma_{\mu}-i\left(F_{2}\left(q^{2}\right)+i F_{E}\left(q^{2}\right) \gamma_{5}\right) \sigma_{\mu \nu} q^{\nu}\right. \\
& \left.+F_{A}\left(q^{2}\right)\left(q^{2} \gamma_{\mu}-\not q q_{\mu}\right) \gamma_{5}\right] \nu,
\end{aligned}
$$

where $q_{\mu}$ is the four momentum transfer; $q^{2}=q_{\mu} q^{\mu}$; $\mathscr{q}=q_{\mu} \gamma^{\mu}$; and $\gamma_{\mu}, \gamma_{5}$, and $\sigma_{\mu \nu}$ are the standard Dirac matrices. The form factors near the $q^{2} \rightarrow 0$ limit yield the definitions of millicharge $\delta_{Q}=F_{1}(0)$, magnetic moment $\mu_{\nu}=F_{2}(0)$, electric dipole moment $d_{\nu}=F_{E}(0)$, and anapole moment $a_{\nu}=F_{A}(0)$ of a neutrino.

There are some important theory aspects to point out here: First, consider neutrino scattering off a free electron, the differential cross section $d \sigma / d T$ with respect to neutrino energy deposition $T$, scales as $T^{-2}$ for the millicharge interaction [13,22], $T^{-1}$ for the magnetic and electric dipole moment interaction [23,24], and $T^{0}$ [25] for the anapole moment and charge-radius-squared interaction. This implies enhanced sensitivities to $\delta_{Q}, \mu_{\nu}$, and $d_{\nu}$ for detectors with low thresholds. Also note that the interactions with $a_{\nu}$ and $\left\langle r_{\nu}^{2}\right\rangle$ have the same contact forms as the low energy weak interactions, so they can be effectively included by modifying the neutrino weak coupling strengths.

Second, as solar neutrinos are ultrarelativistic, that is, $E_{\nu} \gg m_{\nu}$, it is a good approximation to set $m_{\nu}=0$ in scattering. At this limit, a neutrino helicity eigenstate is also a chirality eigenstate. By the identities

$\bar{\nu}_{R} \sigma_{\mu \nu} \nu_{L}=-\bar{\nu}_{R} \sigma_{\mu \nu} \gamma_{5} \nu_{L}, \quad \bar{\nu}_{L} \gamma_{\mu} \nu_{L}=-\bar{\nu}_{L} \gamma_{\mu} \gamma_{5} \nu_{L}$,

one sees that the $\mu_{\nu}$ and $d_{\nu}$ interactions are not distinguishable, nor are the $a_{\nu}$ and $\left\langle r_{\nu}^{2}\right\rangle$ interactions. They will appear in scattering amplitudes as linear combinations $\mu_{\nu}-i d_{\nu}$ and $\left\langle r_{\nu}^{2}\right\rangle-6 a_{\nu}$.

Third, because neutrinos oscillate and the final neutrino type is not observed in the detector setup that we are discussing, the general EM moments of neutrinos should be written in a $3 \times 3$ matrix form to accommodate the possible transition EM moments. The matrixes of the moments involve neutrinos of different types in the incoming and outgoing states, in addition to the static EM moments, which are the diagonal matrix elements. Thus for solar neutrinos, the magnetic moment probed at the detector end is actually an "effective" one which appears in the cross section in a squared form as

$$
\left|\mu_{\nu_{\mathrm{S}}}^{\mathrm{eff}}\right|^{2}=\sum_{f}\left|\sum_{i} A_{i e}\left(E_{\nu}, L\right)\left(\mu_{f i}-i d_{f i}\right)\right|^{2},
$$

where $f$ and $i$ are the mass eigenstate indices for the outgoing and incoming neutrinos at the point of the neutrino EM interaction caused by the transition magnetic and electric dipole moments $\mu_{f i}$ and $d_{f i}$, respectively $[10,12,26]$. The amplitude $A_{i e}\left(E_{\nu}, L\right)$ describes how a solar neutrino, which is an electron neutrino $\nu_{e}$ at birth, oscillates to a mass eigenstate $\nu_{i}$ through the in-medium oscillation in the solar interior and the subsequent vacuum oscillation while traversing from the surface of the Sun to the Earth. The summation over $i$ gives the total transition amplitude 
TABLE I. Selected bounds on the effective magnetic moments and millicharges of neutrinos. Different methods and sources are used in this list, including direct detection of reactor, solar, and accelerator neutrinos, and indirect inferences from astrophysical observations and theory. More can be found in Refs. $[9,10]$

\begin{tabular}{lcll}
\hline \hline Property & Bound & \multicolumn{1}{c}{ Method } & \multicolumn{1}{c}{ Reference } \\
\hline$\mu_{\nu}^{\text {eff }}$ & 2.9 & Reactor $\bar{\nu}_{e}$ & Beda et al. (2013) [11] \\
$\left(\times 10^{-11} \mu_{B}\right)$ & 2.8 & Solar neutrino & Agostini et al. (2017) [12] \\
& 6.8 & Accelerator $\nu_{e}+e, \nu_{\mu}+e$ & Auerbach et al. (2001) [27] \\
& 0.3 & Red giants luminosity & Raffelt (1999) [28] \\
& $0.11-0.27$ & $\nu_{L} \rightarrow \nu_{R}$ in SN 1987A & Kuznetsov et al. (2009) [29] \\
$\delta_{Q}^{\text {eff }}$ & 1.5 & Reactor $\bar{\nu}_{e}$ & Studenikin et al. (2014) [13] \\
$\left(\times 10^{-12} e_{0}\right)$ & 0.02 & Red giant luminosity & Raffelt (1999) [28] \\
& 0.002 & SN 1987A & Barbiellini and Cocconi (1987) [30] \\
& $3 \times 10^{-9}$ & Neutrality of matter & Raffelt (1999) [28] \\
\hline \hline
\end{tabular}

(squared to give probability), and the summation over $f$ indicates the incoherent sum over different final states. Similar procedures should be applied to effective millicharge $\delta_{Q_{\mathrm{S}}}^{\text {eff }}$ and charge radius squared $\left\langle r_{\nu_{\mathrm{S}}}^{2}\right\rangle^{\text {eff }}$. Note that these effective moments depend on neutrino energy $E_{\nu}$ and the Sun-Earth distance $L$. But in practice, they can be approximated as constants $[10,12]$. In this work, we only consider how effective moments are constrained by experiments but not their composition and their dependence on neutrino oscillation parameters.

In Table I we list several stringent upper bounds on neutrino's effective magnetic moments and millicharges using different methods and sources. The bounds indirectly inferred from astrophysical observations and theory can be much tighter than the ones from direction detection; however, they generally depend on the underlying astrophysical models. In this article, the discovery potential of xenon detectors will be benchmarked against the current bounds set by direct detection.

The majority of solar neutrinos comes from two sources which make up about $98 \%$ of the total solar neutrino flux: the proton-proton ( $\mathrm{pp}$ ) fusion, $p+p \rightarrow d+e^{+}+\nu_{e}$, which produces a continuous spectrum with $E_{\nu}$ from 0 to $420 \mathrm{keV}$ [31], and the electron capture by ${ }^{7} \mathrm{Be}$, ${ }^{7} \mathrm{Be}+e^{-} \rightarrow{ }^{7} \mathrm{Li}+\nu_{e}$, which produces two discrete spectral lines at $862 \mathrm{keV}$ and $384 \mathrm{keV}$ with branching ratios $89.6 \%$ and $10.4 \%$. Their fluxes on the Earth's surface are [32,33]

$$
\begin{aligned}
\phi_{\mathrm{pp}} & =5.98 \times 10^{10} \mathrm{~cm}^{-2} \mathrm{~s}^{-1}, \\
\phi_{7_{\mathrm{Be}}} & =5.00 \times 10^{9} \mathrm{~cm}^{-2} \mathrm{~s}^{-1} .
\end{aligned}
$$

Compared with experiments using germanium detectors to probe the EM moments of reactor antineutrinos, the solar neutrino flux is smaller by $2-3$ orders of magnitude. However, ton-scale xenon detectors can make up this deficiency by target mass, as typical germanium detectors only operate at the kilogram scale.

Both pp and ${ }^{7} \mathrm{Be}$ neutrinos have rather low energies. They cannot deposit observable energy at the kilo-electron-volt
$(\mathrm{keV})$ or sub-keV scale by scattering off the whole atom or the atomic nucleus elastically. Therefore, the most effective channel to probe the EM moments of solar neutrinos is to ionize the xenon atom

$$
\nu_{\mathrm{S}}\left(\mathrm{pp},{ }^{7} \mathrm{Be}\right)+\mathrm{Xe} \underset{\text { (weak) }}{\stackrel{\mathrm{EM}}{\longrightarrow}} \nu_{\mathrm{S}}+\mathrm{Xe}^{+}+e^{-},
$$

which produces an electron recoil (ER) with a few tens of $\mathrm{eV}$ to a few tens of $\mathrm{keV}$ in energy. Apparently, the weak scattering is the main background that limits the discovery potential. However, xenon detectors cannot differentiate an ER event from a nuclear recoil (NR) event below certain energy, currently around $1.4 \mathrm{keV} \mathrm{[1].}{ }^{1}$ Therefore, the NR events caused by coherent neutrino-nucleus scattering (CNNS) from the ${ }^{8} \mathrm{~B}$ neutrinos

$$
\nu_{\mathrm{S}}\left({ }^{8} \mathrm{~B}\right)+\mathrm{Xe} \underset{(\mathrm{CNNS})}{\longrightarrow} \nu_{\mathrm{S}}+\mathrm{Xe},
$$

should also be considered as a background.

The formalism for calculating the differential cross sections of neutrino-atom ionization of Eq. (5) is documented in detail in Ref. [17]. The formalism for CNNS of Eq. (6) can be found in Ref. [34]. We only reiterate the importance of atomic structure in these calculations and highlight some key features of our results.

To properly handle the structure of a heavy atom like xenon, several improvements on the conventional mean field approach, the Hartree-Fock approximation, have to be implemented. Our approach in this work is the RRPA [18-21], which builds in the leading relativistic correction and residual two-electron correlation by using the Dirac equation and the RPA equation, respectively.

\footnotetext{
${ }^{1}$ Reference [1] assigns thresholds of $1.4 \mathrm{keV}_{\mathrm{ER}}$ and $4.9 \mathrm{keV}_{\mathrm{NR}}$ to detect ER and NR signals, respectively. The NR threshold is higher because of the quenching effect.
} 
Our computation in this work is very similar to a previous one for a germanium detector, which is fully documented in Ref. [17]. The only major difference is that xenon, being closed shell, does not need the multiconfiguration reference state treatment as the open-shell germanium. For completeness, we briefly recapitulate the main steps here.

First, the ground state wave function is calculated by the Dirac-Fock package [35]. Then the excited states due to an external perturbation are solved by the atomic RRPA equations [18]. The general formulation of the RRPA equation for neutrino-atom scattering can be found in Sec. III. A of Ref. [17] and the computation of the scattering amplitude in Sec. III. B. The formulation of scattering cross sections due to neutrino EM moments is given in Sec. II of Ref. [17], and the explicit expressions for the magnetic moment and millicharge interaction are given in Eqs. (17) and (20), respectively. It should be mentioned that we exclusively use the spherical basis. At low momentum transfer, this basis, facilitated by angular momentum and parity selection rules, is very efficient. However, at higher momentum transfer, the convergence gradually becomes numerically challenging.

This approach was benchmarked by the photoxenon absorption process shown in Fig. 1 of Ref. [8]. The calculation agrees with the experimental data [36-40] at a few percent level between $100 \mathrm{eV}$ to $30 \mathrm{keV}$, except near atomic ionization thresholds or "edges." A photoabsorption edge is where the cross section reaches a local maximum. So a small mismatch between the experimentally measured and the theoretically calculated energy edges leads to a large cross section difference around it. However, this mismatch is largely removed by considering the finite energy resolution of a detector. The theory error is conservatively estimated to

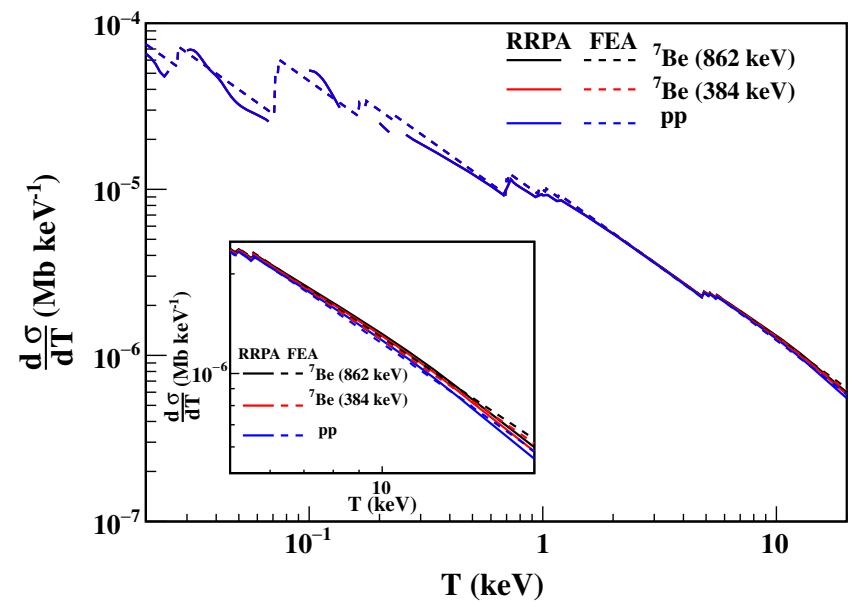

FIG. 1. $\quad \nu$-Xe differential cross sections through the neutrino magnetic moment interaction for the ${ }^{7} \mathrm{Be}\left(862 \mathrm{keV}\right.$, in black), ${ }^{7} \mathrm{Be}$ (384 keV, in blue), and flux averaged pp (in red) solar neutrinos, respectively. These curves largely overlap below $5 \mathrm{keV}$. The difference is highlighted in the inset. The dashed curves are the results of FEA. be $5 \%$ in this range. Between 70 and $100 \mathrm{eV}$, the large peak at around $100 \mathrm{eV}$ indicates strong correlations. Due to slow convergence of the computation in this range, only a few points were obtained. The largest disagreement is seen between 40 and $70 \mathrm{eV}$ but still within $30 \%$. These discrepancies of our calculations and experimental data roughly characterize the theoretical error. Furthermore, photoabsorption is a real-photon process but neutrino EM scattering is a virtual-photon one. Therefore, only the on-shell atomic response is benchmarked.

In Fig. 1, the $\nu$-Xe differential cross sections through the neutrino magnetic moment interaction are shown. Contributions from the ${ }^{7} \mathrm{Be}$ line spectrum at $862 \mathrm{keV}$ and $384 \mathrm{keV}$, and the flux averaged pp neutrinos all have very similar $T$ dependence. We also show the result using the stepping free electron approximation (FEA):

$$
\frac{d \sigma^{(i)}}{d T}=\sum_{i=1}^{Z} \theta\left(T-B_{i}\right) \frac{d \sigma_{0}^{(i)}}{d T} .
$$

This is done by weighting the scattering cross section of a neutrino and a free electron, $d \sigma_{0} / d T$, with the number of electrons that can be ionized by an energy deposition of $T$, with $\theta$ the step function and $B_{i}$ the binding energy of the $i$ th electron. FEA is shown to be a good approximation for this process from a sum rule analysis [41-43]. This conclusion was confirmed numerically by the ab initio calculation with Ge in Ref. [16] and with Xe in this work. However, the $a b$ initio result is consistently smaller than that of FEA in Ge but not in Xe here. This can be traced back to the approximation used in the sum rule method which keeps only the leading single electron operator $J^{0}=e^{\dagger} e$ in the nonrelativistic expansion of the electromagnetic current. The $J^{i}$ current $(i=1,2,3)$, which is suppressed by the electron velocity, and higher dimensional operators such as $\delta J^{0}=\left(e^{\dagger} e\right)^{2}$, are not included in the analysis. However, from an effective field theory point of view, all operators of the same quantum numbers as $J^{0}$ and $J^{i}$ can appear after the high energy mode in the nonrelativistic effective field theory is "integrated out." For example, the one electron operator $e^{\dagger} e$ can exchange one high energy photon with another electron. After integrating out the high energy photon, the $\delta J^{0}$ operator appears. With just the $J^{0}$ operator, one can show that FEA yields an upper bound. However, the inclusion of $\delta J^{0}$ could change this conclusion. A similar conclusion was reached in Ref. [43] that electron correlations could modify the sum rule analysis of [41]. Indeed, our result becomes bigger than FEA near $100 \mathrm{eV}$ where a large peak in photoabsorption is observed in Fig. 1 of Ref. [8]. This peak should come from large electron correlations since the nearest threshold is still $30 \mathrm{eV}$ away.

Analogously, the $\nu$-Xe differential cross sections through the neutrino millicharge interaction are shown in Fig. 2. The millicharge interaction has a sharper $T$ dependence than the neutrino magnetic moment interaction which is 


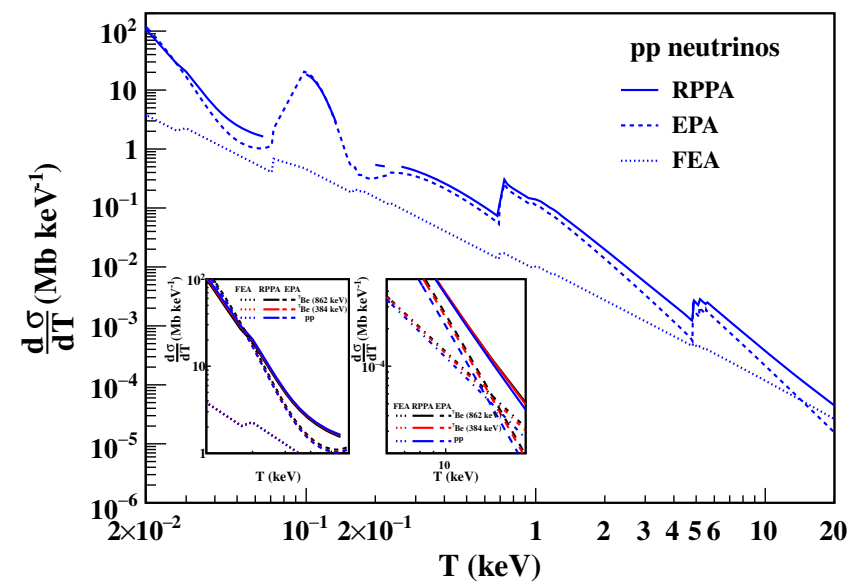

FIG. 2. $\nu$-Xe differential cross sections through the neutrino millicharge interaction for the ${ }^{7} \mathrm{Be}\left(862 \mathrm{keV}\right.$, in black), ${ }^{7} \mathrm{Be}$ (384 keV, in blue), and flux averaged pp (in red) solar neutrinos, respectively. These curves largely overlap. The difference is highlighted in the insets. The FEA and EPA results are also shown.

also seen in scattering over a free electron. It has been shown in the previous study that at low energies, the equivalent photon approximation (EPA), which relates the ionization cross section to the one of photoabsorption, works well in the Ge ionization by the neutrino millicharge interaction [14]. While at energies higher than all the electron binding energies, electron binding is no longer important and the result approaches the FEA curve [14]. These features are also seen in the Xe case here.

\section{RESULTS AND DISCUSSIONS}

The differential count rates as functions of neutrino energy deposition, $d R(T) / d T$, are obtained by convoluting the differential cross sections $d \sigma\left(T, E_{\nu}\right) / d T$ with the incident solar neutrino energy spectrum $d \phi\left(E_{\nu}\right) / d E_{\nu}$,

$$
\frac{d R(T)}{d T}=\frac{N_{0} \Delta t}{A} \int d E_{\nu} \frac{d \sigma\left(T, E_{\nu}\right)}{d T} \frac{d \phi\left(E_{\nu}\right)}{d E_{\nu}},
$$

where the unit is fixed as events per ton per year per $\mathrm{keV}$, $N_{0}=6.02 \times 10^{29}, A$ is the atomic mass of the atom, and $\Delta t=1$ year.

We have shown relevant solar neutrino xenon scattering processes using the current best upper limits on neutrino EM moments as inputs for the effective EM moments of solar neutrinos in Fig. 3: $\delta_{Q_{\mathrm{S}}}^{\mathrm{eff}}=1.5 \times 10^{-12} e_{0}, \mu_{\nu_{\mathrm{S}}}^{\mathrm{eff}}=2.8 \times 10^{-11} \mu_{\mathrm{B}}$, and $\left\langle r_{\nu_{\mathrm{S}}}^{2}\right\rangle^{\text {eff }}=3.3 \times 10^{-32} \mathrm{~cm}^{2}$. The rates are with one ton-year exposure.

The red curve is for the electron recoil from atomic ionization $\left(\nu+A \rightarrow \nu+A^{+}+e^{-}\right)$through the standard weak scattering which is considered as a background in this work. In the range of $T$ considered, major contributions are from the pp and ${ }^{7} \mathrm{Be}$ neutrinos. Note that charged-current

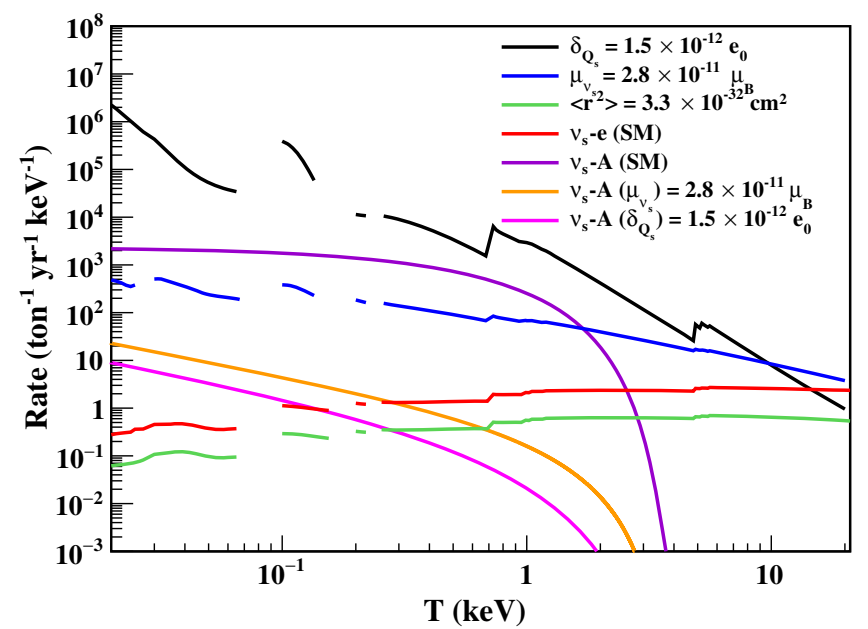

FIG. 3. Electron and nuclear recoil event rates from solar neutrino scattering off xenon. Electron recoil channels: neutrino atomic ionization through weak interaction (red), neutrino millicharge (black, scales as $\delta_{Q_{S}}^{2}$ ), magnetic moment (blue, scales as $\mu_{\nu_{S}}^{2}$ ), and charge radius (green, scales as $\left\langle r^{2}\right\rangle$, from interference with the weak interaction contribution). Nuclear recoil channels: neutrino nucleus coherent scatterings through weak interaction (purple), neutrino magnetic moment (golden, scales as $\mu_{\nu_{S}}^{2}$ ), and neutrino millicharge (magenta, scales as $\delta_{Q_{S}}$, from interference with the weak interaction contribution). $e_{0}$ is the electron charge and $\mu_{B}$ is Bohr magneton. Some lines are broken due to slow convergence of the RRPA calculation in the broken areas.

interaction is not flavor blind $\left(\sigma_{\nu_{\mu}}=\sigma_{\nu_{\tau}} \neq \sigma_{\nu_{e}}\right)$ and will depend on solar neutrino oscillations. The result presented here is based on the same computation scheme as the one of Ref. [8] with neutrino oscillations included but with improved error estimation. The differential cross section at large $T$ is slightly increased, but still smaller than the calculation [7] based on free electron scattering by $19 \%$.

The purple curve is for the nuclear recoil from neutrinonucleus coherent scattering $(\nu+A \rightarrow \nu+A)$ which is also considered as a background here since ER and NR events cannot be distinguished at low energies (current below $1.4 \mathrm{keV})$. At $T>0.01 \mathrm{keV}_{\mathrm{NR}}$, it is dominated by the ${ }^{8} \mathrm{~B}$ neutrinos, because pp and ${ }^{7} \mathrm{Be}$ neutrinos are not energetic enough. The process only involves the flavor-blind neutralcurrent interaction so it is independent of solar neutrino oscillations. The event rate presented here is based on the standard formula for neutrino-nucleus coherent scattering, quenching factor $\mathrm{Q}_{f}=1$ (i.e., no quenching), and the coherency is more than 0.9 in the range of $T$ [34].

$\nu$-Xe EM ionization event rates are presented by black (millicharge), blue (magnetic moment), and green curves (charge radius squared), respectively. The result suggests that if the detector has good sensitivity to the weak interaction (red curve) background, then the current bound on neutrino effective millicharge can be further improved. The improvement on the current bound of neutrino effective magnetic moment is also possible especially with 
TABLE II. Summary of experimental limits at $90 \%$ C.L. on the effective EM moments: $\mu_{\nu_{\mathrm{S}}}^{\text {eff }}, \delta_{Q_{\mathrm{S}}}^{\text {eff }}$, and $\left\langle r_{\nu_{\mathrm{S}}}^{2}\right\rangle^{\text {eff }}$ of solar neutrinos, assuming an energy resolution from the XENON100 experiment [46].

\begin{tabular}{|c|c|c|c|c|c|c|}
\hline \multirow[b]{2}{*}{ Experiment } & \multirow[b]{2}{*}{$\begin{array}{l}\text { Exposure } \\
\text { (ton-year) }\end{array}$} & \multirow[b]{2}{*}{$\begin{array}{c}\text { Threshold } \\
\left(\mathrm{keV}_{\mathrm{ER}}\right)(\mathrm{S} 1+\mathrm{S} 2)\end{array}$} & \multirow[b]{2}{*}{$\begin{array}{c}\text { Background level } \\
\left(\mathrm{kg}^{-1} \mathrm{keV}^{-1} \text { day }^{-1}\right)\end{array}$} & \multicolumn{3}{|c|}{ Upper bounds at $90 \%$ C.L. } \\
\hline & & & & $\begin{array}{c}\mu_{\nu_{\mathrm{S}}}^{\text {eff }} \\
\left(\times 10^{-11} \mu_{\mathrm{B}}\right) \\
\end{array}$ & $\begin{array}{c}\delta_{Q_{\mathrm{S}}}^{\mathrm{eff}} \\
\left(\times 10^{-12} e_{0}\right) \\
\end{array}$ & $\begin{array}{c}\left\langle r_{\nu_{\mathrm{S}}}^{2}\right\rangle{ }^{\mathrm{eff}} \\
\left(\times 10^{-30} \mathrm{~cm}^{2}\right) \\
\end{array}$ \\
\hline XENON-10 [47] & $8.67 \times 10^{-2}$ & 2.0 & 1.1 & 348.74 & 65.45 & 158.86 \\
\hline XENON-100 [48] & $2.1 \times 10^{-2}$ & 5.0 & $5.3 \times 10^{-3}$ & 35.13 & 13.03 & 11.20 \\
\hline PandaX-II [49] & $7.4 \times 10^{-2}$ & 1.2 & $2.7 \times 10^{-3}$ & 15.46 & 2.06 & 8.13 \\
\hline XENON-1T [50] & 1.0 & 1.4 & $2.24 \times 10^{-4}$ & 4.51 & 0.64 & 2.31 \\
\hline Projected LZ [51] & 15.34 & $1.5 / 0.5$ & $42.7 \times 10^{-5}$ & $1.85 / \sim 1$ & $0.28 / \sim 0.01$ & 0.93 \\
\hline Projected DARWIN [44] & 14.0 & 2.0 & $2.0 \times 10^{-5}$ & 1.27 & 0.24 & 0.58 \\
\hline $\mathrm{LZ}+{ }^{51} \mathrm{Cr}$ source $[52]$ & 0.82 & 2.0 & $4.27 \times 10^{-5}$ & 0.61 & 0.156 & 0.0276 \\
\hline
\end{tabular}

$T>2 \mathrm{keV}$. Below $2 \mathrm{keV}$ the NR background from neutrino-nucleus coherent scattering (purple curve) needs to be subtracted, which might be a challenge.

We also study the possible signal of $\nu$-Xe EM interaction through the NR process. The golden curve is neutrinonucleus coherent scattering through neutrino magnetic moment using

$\frac{d \sigma_{\mu}(\nu A \rightarrow \nu A)}{d T_{\mathrm{NR}}}=\frac{\pi \mu_{\nu}^{2} \alpha^{2} Z^{2}}{m_{e}^{2}}\left(\frac{1}{T_{\mathrm{NR}}}-\frac{1}{E_{\nu}}+\frac{T_{\mathrm{NR}}}{4 E_{\nu}^{2}}\right) F_{V}^{2}\left(q^{2}\right)$,

where $Z$ is the atomic number, $m_{e}$ is the electron mass coming from the definition of Bohr magneton, $F_{V}\left(q^{2}\right)$ with normalization $F_{V}(0)=1$ is the nuclear isoscalar vector form factor for xenon which is an isoscalar nucleus, and the on-shell condition for the nucleus fixes $q^{2}=-2 M_{A} T_{\mathrm{NR}}$ [24]. The effect is much smaller than coherent scattering through weak interaction and is also much smaller than ER through neutrino magnetic moment interaction. A similar NR process through the neutrino millicharge can interfere with the weak interaction process:

$$
\begin{aligned}
\frac{d \sigma_{\delta_{Q}}(\nu A \rightarrow \nu A)}{d T_{\mathrm{NR}}}= & \frac{M_{A} G_{F}^{2}}{4 \pi}\left(1-\frac{M_{A} T_{\mathrm{NR}}}{2 E_{\nu}^{2}}\right) \\
& \times\left(2 A \sin ^{2} \theta_{W}+x\right)^{2} F_{V}^{2}\left(q^{2}\right), \\
x= & \frac{2 \sqrt{2} \pi \alpha Z \delta_{Q}}{G_{F} M_{A} T_{\mathrm{NR}}},
\end{aligned}
$$

where $G_{F}$ is the Fermi coupling constant, $\theta_{W}$ is the Weinberg angel, and $M_{A}$ is the nuclear mass. The current $\delta_{Q}$ bound makes the interference term (the $x$ term) much bigger than the $x^{2}$; hence we present the $x$ term as the magenta curve.

To put the above naive (and optimistic, too) prospect on a more realistic ground, one needs to take detector specifications into account. All relevant backgrounds can be categorized into external and intrinsic ones. The external background comes from radioactive contamination in detector construction materials which can be reduced considerably by target fiducialization. However, this selfshielding does not work for the intrinsic background from ${ }^{85} \mathrm{Kr},{ }^{222} \mathrm{Rn}$, and double beta decays of ${ }^{136} \mathrm{Xe}$. A potential background for neutrino-electron interactions comes from these components. Based on earlier studies [44,45], the total ER background distribution is assumed to be flat below $20 \mathrm{keV}$. The upper limits on the various neutrino electromagnetic parameters are derived under the assumption that the predicted signal cannot exceed the measured background rate. The energy resolution for xenon detectors is adopted from Ref. [46].

It is also worth emphasizing that the distinguishing power of NR and ER for liquid Xe detectors relies on the S1 (prompt scintillation) signal. In the absence of the S1 signal, the electromagnetic background contamination increases significantly due to losing $z$-coordinate reconstruction from the time difference of $\mathrm{S} 2 / \mathrm{S} 1$ and particle identification based on the $\mathrm{S} 2 / \mathrm{S} 1$ signal ratio. More specifically, although the S2 energy threshold is lower than S1 and can be pushed to sub-keV due to electroluminescence amplification, also the ambient background can be minimized by using a smaller fiducial mass; however, the NR background from neutrino-nucleus coherent scattering becomes a major issue in the absence of discrimination between NR and ER events in the S2-only analysis. Therefore, we will only present constraints from the $\mathrm{S} 1+\mathrm{S} 2$ combined analyses.

In Table II, we show the key detector parameters for LXe detectors and their sensitivity on various solar neutrino EM moments for existing experiments: XENON-10 [47], XENON-100 [48], PandaX-II [49], XENON-1T [50]; and future experiments: LZ [51] and DARWIN [44]. It is interesting that with one ton-year exposure, XENON-1T can improve the $\delta_{Q_{\mathrm{S}}}^{\text {eff }}$ constraint by a factor of 2 . With LZ and DARWIN, the projected improvement on $\delta_{Q_{S}}^{\text {eff }}$ is around 7 times smaller than the current bound, while the projected 
improvement on $\mu_{\nu_{\mathrm{S}}}^{\mathrm{eff}}$ is around a factor of 2. To further motivate future experimental effort, if $\mathrm{LZ}$ can keep the same background level and push the ER threshold to $0.5 \mathrm{keV}_{\mathrm{ER}}$ with $\mathrm{S} 1+\mathrm{S} 2$, then limits of $\delta_{Q_{\mathrm{S}}}^{\text {eff }} \sim 10^{-13} e_{0}$ (about 1 order smaller than the current bound) and $\mu_{\nu_{\mathrm{S}}}^{\text {eff }} \sim 10^{-11} \mu_{B}$ (about 3 times smaller than the current bound) can be achieved.

For the effective charge radius squared, we see the constraints in Table II are no better than the existing bound. This is because the associated cross section is not enhanced relative to the weak interaction background at low energy. Hence the best constraint is in fact from the TEXONO experiment using $\mathrm{MeV}$ reactor neutrinos [15].

Additionally in the last column of Table II, we consider an unconventional setup suggested in Ref. [52] that places a strong artificial radioactive source, $5 \mathrm{MCi}$ of ${ }^{51} \mathrm{Cr}, 1 \mathrm{~m}$ below the fiducial 6 ton volume of the LZ detector. Assuming a 50-day exposure (note that ${ }^{51} \mathrm{Cr}$ has a short half-life of 27.7 days), the time and shape average neutrino flux is about $3.2 \times 10^{11} / \mathrm{cm}^{2} / \mathrm{sec}$, which is more intense than the solar neutrino flux. As a result, the constraints on all neutrino EM moments can be much improved. ${ }^{2}$ However, this setup would require turning a ton-scale xenon detector from a dark matter search mode to a neutrino detection mode, at least for a certain period of time.

\section{SUMMARY}

In this work, we study the potential of using current and next-generation xenon detectors in constraining exotic electromagnetic moments of neutrinos with low energy solar $\mathrm{pp}$ and ${ }^{7} \mathrm{Be}$ sources. The cross sections of neutrinoatom scattering due to these electromagnetic neutrinoelectron interactions are calculated by the relativistic random phase approximation which is a state-of-the-art

\footnotetext{
${ }^{2}$ We have checked explicitly with Ref. [52] that by assuming the same 100-day exposure, a smaller background level at $1.66 \times 10^{-5} \mathrm{~kg}^{-1} \mathrm{keV}^{-1} \mathrm{day}^{-1}$, and the free electron approximation for neutrino-atom scattering, the same upper limit on the neutrino magnetic moment at $4.9 \times 10^{-12} \mu_{\mathrm{B}}$ is obtained.
}

$a b$ initio approach to properly take atomic many-body physics into account. Limits are derived using current experimental data with subton-year exposure. Projected sensitivity is also estimated assuming future detector specifications.

It is interesting that with one ton-year exposure, XENON-1T can improve the effective millicharge constraint by a factor of 2. With LZ and DARWIN, the projected improvement on the effective millicharge is around 7 times smaller than the current bound, while the projected improvement on the effective magnetic moment is around a factor of 2. To further motivate future experimental effort, if LZ can keep the same background level and push the ER threshold to $0.5 \mathrm{keV}_{\mathrm{ER}}$ with $\mathrm{S} 1+\mathrm{S} 2$, then limits of $\delta_{Q_{\mathrm{S}}}^{\text {eff }} \sim 10^{-13} e_{0}$ (1 order smaller than the current bound) and $\mu_{\nu_{\mathrm{S}}}^{\text {eff }} \sim 10^{-11} \mu_{B}$ ( 3 times smaller than the current bound) can be achieved.

As for the limit on the effective charge radius of neutrinos, using low energy solar neutrinos has no advantage over using $\mathrm{MeV}$ reactor neutrinos. As for contributions from additional electromagnetic neutrino-nucleus interactions, the resulting nuclear recoil signals are swamped by the background due to coherent neutrino-nucleus scattering.

We also consider the setup of placing a strong neutrino source ${ }^{51} \mathrm{Cr}$ nearby a ton-scale xenon detector. As the neutrino flux is larger than the solar one, all neutrino EM properties can be much improved. However, this would require running a xenon detector in the neutrino detection mode instead of the dark matter search mode.

\section{ACKNOWLEDGMENTS}

This work is supported in part by the Ministry of Science and Technology, Taiwan, under Grants No. 1042112-M-259-004-MY3, No. 105-2112-M-002-017-MY3, No. 107-2811-M-002-3063, No. 107-2811-M-002-3097, No. 107-2112-M-259-002, and No. 107-2119-M-001-028MY3; Grant No. 2019-20/ECP-2 from the National Center for Theoretical Sciences; and the Kenda foundation.
[1] E. Aprile et al. (XENON Collaboration), Phys. Rev. Lett. 121, 111302 (2018).

[2] X. Cui et al. (PandaX-II), Phys. Rev. Lett. 119, 181302 (2017).

[3] E. Aprile et al. (XENON Collaboration), J. Cosmol. Astropart. Phys. 04 (2016) 027.

[4] D. S. Akerib et al. (LZ Collaboration), arXiv:1509.02910.

[5] J. Aalbers et al. (DARWIN Collaboration), arXiv:1606.07001.

[6] J. Billard, L. Strigari, and E. Figueroa-Feliciano, Phys. Rev. D 89, 023524 (2014).
[7] L. Baudis, A. Ferella, A. Kish, A. Manalaysay, T. M. Undagoitia, and M. Schumann, J. Cosmol. Astropart. Phys. 01 (2014) 044.

[8] J.-W. Chen, H.-C. Chi, C.-P. Liu, and C.-P. Wu, Phys. Lett. B 774, 656 (2017).

[9] M. Tanabashi et al. (Particle Data Group), Phys. Rev. D 98, 030001 (2018).

[10] C. Giunti and A. Studenikin, Rev. Mod. Phys. 87, 531 (2015). 
[11] A. G. Beda, V. B. Brudanin, V. G. Egorov, D. V. Medvedev, V.S. Pogosov, E. A. Shevchik, M. V. Shirchenko, A. S. Starostin, and I. V. Zhitnikov, Phys. Part. Nucl. Lett. 10, 139 (2013).

[12] M. Agostini et al. (Borexino Collaboration), Phys. Rev. D 96, 091103(R) (2017).

[13] A. I. Studenikin, Eur. Phys. Lett. 107, 21001 (2014).

[14] J.-W. Chen, H.-C. Chi, H.-B. Li, C.-P. Liu, L. Singh, H. T. Wong, C.-L. Wu, and C.-P. Wu, Phys. Rev. D 90, 011301(R) (2014).

[15] M. Deniz et al. (TEXONO Collaboration), Phys. Rev. D 81, 072001 (2010); 82, 033004 (2010).

[16] J.-W. Chen, H.-C. Chi, K.-N. Huang, C.-P. Liu, H.-T. Shiao, L. Singh, H. T. Wong, C.-L. Wu, and C.-P. Wu, Phys. Lett. B 731, 159 (2014).

[17] J.-W. Chen, H.-C. Chi, K.-N. Huang, H.-B. Li, C.-P. Liu, L. Singh, H. T. Wong, C.-L. Wu, and C.-P. Wu, Phys. Rev. D 91, 013005 (2015).

[18] W. R. Johnson and C. D. Lin, Phys. Rev. A 20, 964 (1979).

[19] W. R. Johnson and K. T. Cheng, Phys. Rev. A 20, 978 (1979).

[20] K.-N. Huang, W. R. Johnson, and K. T. Cheng, Phys. Rev. Lett. 43, 1658 (1979).

[21] K.-N. Huang, W. R. Johnson, and K. T. Cheng, At. Data Nucl. Data Tables 26, 33 (1981).

[22] S. Gninenko, N. Krasnikov, and A. Rubbia, Phys. Rev. D 75, 075014 (2007).

[23] A. V. Kyuldjiev, Nucl. Phys. B243, 387 (1984).

[24] P. Vogel and J. Engel, Phys. Rev. D 39, 3378 (1989).

[25] M. J. Musolf and B. R. Holstein, Phys. Rev. D 43, 2956 (1991).

[26] J. F. Beacom and P. Vogel, Phys. Rev. Lett. 83, 5222 (1999).

[27] L. B. Auerbach et al., Phys. Rev. D 63, 112001 (2001).

[28] G. G. Raffelt, Stars as Laboratories for Fundamental Physics (University Chicago Press, Chicago, 1996), Sect. 7.5.

[29] A. V. Kuznetsov, N. V. Mikheev, and A. A. Okrugin, Int. J. Mod. Phys. A 24, 5977 (2009).

[30] G. Barbiellini and G. Cocconi, Nature (London) 329, 21 (1987).

[31] J. N. Bahcall, A. M. Serenelli, and S. Basu, Astrophys. J. 621, L85 (2005).
[32] A. M. Serenelli, W. C. Haxton, and C. Pena-Garay, Astrophys. J. 743, 24 (2011).

[33] E. G. Adelberger et al., Rev. Mod. Phys. 83, 195 (2011).

[34] S. Kerman, V. Sharma, M. Deniz, H. T. Wong, J.-W. Chen, H. B. Li, S. T. Lin, C.-P. Liu, and Q. Yue (TEXONO Collaboration), Phys. Rev. D 93, 113006 (2016).

[35] J. P. Desclaux, Comput. Phys. Commun. 9, 31 (1975).

[36] J. B. West and J. Morton, At. Data Nucl. Data Tables 22, 103 (1978).

[37] B. L. Henke, E. M. Gullikson, and J. C. Davis, At. Data Nucl. Data Tables 54, 181 (1993).

[38] J. Samson and W. Stolte, J. Electron Spectrosc. Relat. Phenom. 123, 265 (2002).

[39] I. H. Suzuki and N. Saito, J. Electron Spectrosc. Relat. Phenom. 129, 71 (2003).

[40] L. Zheng, M. Cui, Y. Zhao, J. Zhao, and K. Chen, J. Electron Spectrosc. Relat. Phenom. 152, 143 (2006).

[41] M. B. Voloshin, Phys. Rev. Lett. 105, 201801 (2010); 106, 059901(E) (2011).

[42] K. A. Kouzakov and A. I. Studenikin, Phys. Lett. B 696, 252 (2011).

[43] K. A. Kouzakov, A. I. Studenikin, and M. B. Voloshin, Phys. Rev. D 83, 113001 (2011).

[44] L. Baudis, A. Ferella, A. Kish, A. Manalaysay, T. M. Undagoitia, and M. Schumann, J. Cosmol. Astropart. Phys. 01 (2014) 044.

[45] M. Schumann, L. Baudis, L. Bütikofer, A. Kish, and M Selvi, J. Cosmol. Astropart. Phys. 10 (2015) 016.

[46] E. Aprile et al. (XENON100 Collaboration), Astropart. Phys. 35, 573 (2012).

[47] E. Aprile et al. (XENON10 Collaboration), Astropart. Phys. 34, 679 (2011).

[48] E. Aprile et al. (XENON100 Collaboration), Phys. Rev. D 96, 122002 (2017).

[49] C. Fu et al. (Pandax-II Collaboration), Phys. Rev. Lett. 119, 181806 (2017).

[50] E.Aprile et al. (XENON Collaboration), Phys. Rev. Lett. 121, 111302 (2018).

[51] D. S. Akerib et al. (LZ Collaboration), arXiv:1802.06039v1.

[52] P. Coloma, P. Huber, and J. M. Link, J. High Energy Phys. 11 (2014) 042. 